\title{
Prácticas y creencias de enseñanza y estimulación del lenguaje desde la Cultura Mapuche en niños y niñas rurales en la Región de La Araucanía
}

\author{
Teaching practices and beliefs and language stimulation from the Mapuche Culture \\ in rural children in the Araucanía Region
}

\author{
Francisca Fernández Gutiérrez ${ }^{\mathrm{a}, \mathrm{c}}$, Ana María Alarcón Muñoz ${ }^{\mathrm{b}, \mathrm{c}}$ \\ aFacultad de Enfermería. Universidad Andrés Bello. Chile \\ bDepartamento de Salud Pública, Capacitación, Investigación y Gestión para la Salud basada en Evidencias (CIGES). \\ Universidad de La Frontera. Chile \\ 'Enfermera
}

Recibido: 4 de diciembre de 2018; Aceptado: 9 de septiembre de 2019

¿Qué se sabe del tema que trata este estudio?

La valoración de la infancia para la cosmovisión mapuche va más allá de lo pondoestatural, sino que además se cruza con componentes valóricos y culturales.
¿Qué aporta este estudio a lo ya conocido?

El lenguaje es observado como un acto pragmático, donde el habla con sentido será considerada como hito del desarrollo.

\section{Resumen}

El lenguaje es una de las tareas cognitivas más relevantes en el desarrollo infantil cuya adquisición está mediada, entre otros, por factores étnicos y culturales. Objetivo: Caracterizar las prácticas y creencias sobre la enseñanza y estrategias de estimulación del lenguaje de niños/as Mapuche rurales de 0 a 4 años desde una perspectiva intracultural. Sujetos y Método: Estudio cualitativo basado en Teoría Fundamentada que produce una comprensión del problema a partir de las percepciones de los sujetos de investigación. Se utilizaron técnicas etnográficas como observación y notas de campo además de 20 entrevistas en profundidad y 4 grupos focales con cuidadores (madres, padres, abuelos/as), educadores interculturales, asesores culturales y agentes ancestrales de la cultura (longko y machi). Participaron 41 personas Mapuche de los territorios rurales de Ercilla, Curarrehue, Puerto Saavedra, Cholchol y Boroa de la región de La Araucanía/Chile. Resultados: Se obtuvieron 4 dimensiones que caracterizaban las prácticas y creencias en torno a la adquisición y uso del lenguaje en niños/as Mapuche: a) los/as niños/as y el aprendizaje de la lengua representan la mantención de la identidad Mapuche; b) el habla es un proceso concreto y pragmático; c) existen técnicas tradicionales para estimulación del habla, y d) existen dificultades y facilidades para el desarrollo de la lengua indígena en los niños/as pequeños. Conclusiones: La mantención y recuperación de la lengua indígena es un proceso de reforzamiento de la identidad cultural para el pueblo Mapuche, que no ha sido valorado como un patrimonio cultural en la sociedad nacional.
Palabras clave:

Infante; Niño;

Preescolar;

Estimulación del

lenguaje; Grupos

Étnicos

Correspondencia:

María Francisca Fernández Gutiérrez

franciscafernandezg@gmail.com 


\begin{abstract}
Language is one of the most relevant cognitive tasks in child development and its acquisition is mediated, among others, by ethnic and cultural factors. Objective: To characterize the practices and beliefs about language teaching and stimulation strategies of rural Mapuche Children aged between 0 to 4 years from an intracultural perspective. Subjects and Method: Qualitative study based on Grounded Theory, which generates an understanding of the study issue from the perceptions of the research subjects. Ethnographic techniques such as observation and field notes were used, and 20 in-depth interviews and four focus groups were conducted with caregivers (mothers, fathers, and grandparents), intercultural teachers and people with cultural roles (longkos, machis, and intercultural facilitators). 41 Mapuche people from the Ercilla, Curarrehue, Puerto Saavedra, Cholchol and Boroa territories of the Araucanía region, Chile participated. Results: Four dimensions were obtained that characterized the practices and beliefs regarding the acquisition and use of language in Mapuche children: a) Mapuche culture transcends through children and language, b) speech is a concrete and pragmatic process, c) there are traditional techniques for speech stimulation, and d) there are difficulties and easiness for the development of the indigenous language in young children. Conclusions: The preservation and recovery of the indigenous language is a process of reinforcing the cultural identity for the Mapuche people, which has not been valued as a cultural heritage in the national society.
\end{abstract}

Keywords:

Infant, Child,

Preschooler, Language

Development, ethnic

group

\section{Introducción}

En los últimos años, ha surgido con fuerza dentro de las políticas públicas la necesidad de enfocarse en disminuir las desigualdades sociales y enfocarse en grupos vulnerables. En consecuencia, una mejor protección de la infancia es una estrategia para abordar las inequidades y disminuir las brechas del desarrollo humano ${ }^{1}$.

La Comisión Económica para América Latina y el Caribe (CEPAL) identifica a niños/as indígenas como un grupo vulnerable dentro de las sociedades nacionales, y plantea que la infancia indígena debe ser un objetivo y preocupación de los gobiernos en Latinoamérica ${ }^{2}$. En Chile, la región de La Araucanía presenta una alta ruralidad, población dispersa, y conflicto interétnico lo que sitúa a la infancia Mapuche en una zona de riesgo o fragilidad para su desarrollo pleno y potencial. En la región hay aproximadamente 65 mil niños de $0-4$ años, $36 \%$ de ellos pertenece a la etnia Mapuche y el 27,7\% vive en territorios rurales de la Araucanía particularmente en comunidades indígenas ${ }^{3}$.

Diversos estudios señalan la importancia de los factores protectores de la cultura como elementos que contribuyen a cuidar a la infancia en riesgo ${ }^{4,5}$. En Chile estudios sobre crianza de la infancia Mapuche, indican una dinámica de transición entre tradición y cambio; observando una pérdida importante de patrones culturales de estimulación y crianza ${ }^{6,7}$.Se señala que procesos de parentalidad cultural generan estrategias de vigilancia del desarrollo que estimulan el cumplimiento de las necesidades básicas de autonomía y pertenencia en los niños, de modo tal, que convierte a la cultura en un factor de protección del desarrollo infantil ${ }^{8-10}$.
Por otra parte, se indica que el lenguaje es una de las habilidades o destrezas cognitivas más relevantes en el desarrollo psicomotor de la infancia, y que su estimulación gatilla procesos sociocognitivos de alta complejidad ${ }^{11}$. Sin embargo, una perspectiva solamente biopsicosocial de la estimulación y uso del lenguaje no contempla los posibles aportes sociales de la cultura indígena en la adquisición de las destrezas lingüísticas.

Por ello los objetivos que guían este trabajo son: Reconocer la importancia, prácticas y creencias en torno a la adquisición y uso del lenguaje de niños/as Mapuche rurales de 0 a 4 años desde una perspectiva intracultural, y describir las formas de aprendizaje de la lengua indígena y sus formas de uso en los niños/as Mapuche desde la perspectiva de sus cuidadores, educadores y personas con roles culturales ancestrales, en territorios con alta concentración indígena de la región de La Araucanía. Su propósito es reconocer la existencia de formas culturales para el uso y estimulación de la lengua Mapuche (mapudungun) indígena al interior de las comunidades rurales de la Araucanía y promover procesos de pertinencia cultural que apoyen a las familias de la región.

\section{Sujetos y Método}

Estudio cualitativo basado en la teoría fundamentada. Este enfoque teórico metodológico permite desde una comparación y contrastación constante de los datos (transcripciones de entrevistas y grupos focales), generar una teoría emergente del tema de estudio basada en la lectura repetitiva de las narrativas de los sujetos de estudio ${ }^{12}$. La estrategia metodológica de la teoría 
Tabla 1. Distribución muestral por técnica y territorio

\begin{tabular}{|c|c|c|c|c|c|c|}
\hline \multirow{2}{*}{$\begin{array}{l}\text { Territorio } \\
\text { Puerto Saavedra }\end{array}$} & \multirow{2}{*}{$\begin{array}{c}\begin{array}{c}\text { Entrevista } \\
\text { cuidadores }\end{array} \\
2\end{array}$} & \multirow{2}{*}{$\begin{array}{c}\begin{array}{c}\text { Entrevista } \\
\text { docentes }\end{array} \\
1\end{array}$} & \multirow{2}{*}{$\begin{array}{c}\text { Entrevista } \\
\text { agentes ancestrales } \\
1\end{array}$} & \multicolumn{2}{|c|}{$\begin{array}{c}\text { Grupo focal } \\
\text { N\%/participantes }\end{array}$} & \multirow{2}{*}{$\begin{array}{c}\begin{array}{c}\text { Total participante } \\
\text { por territorio }\end{array} \\
9\end{array}$} \\
\hline & & & & 1 & $n=5$ & \\
\hline Kurarrewe & 1 & 0 & 1 & 2 & $\begin{array}{l}{[n=8]} \\
{[n=4]}\end{array}$ & 14 \\
\hline Ercilla & 4 & 2 & 1 & 1 & $n=4$ & 11 \\
\hline Boroa & 2 & 1 & 0 & 0 & $(-)$ & 3 \\
\hline Chol Chol & 2 & 1 & 1 & 0 & $(-)$ & 4 \\
\hline Total participantes & 11 & 5 & 4 & 4 & 21 & 41 \\
\hline
\end{tabular}

fundamentada consiste en una lógica iterativa entre el dato narrativo y su análisis, el que se realiza mediante procesos de codificación.

Los participantes del estudio fueron cuidadores (padres, madres abuelos/as), profesores interculturales bilingües, asesores interculturales, y agentes tradicionales de la comunidad Mapuche (machi [agente terapéutico ancestral], longko [jefe o guía de la comunidad]). La muestra final estuvo compuesta de 41 participantes: 20 entrevistados y 21 participantes en 4 grupos focales (tabla 1).

La recolección de datos se realizó a través de entrevistas en profundidad y grupos focales mediante una pauta compuesta de 6 preguntas o temas que abordaban los objetivos del estudio. Estas técnicas permiten flexibilidad en la respuesta de los participantes ahondando en sus experiencias individuales y personales. Se realizaron 20 entrevistas y 4 grupos focales con la participación de 5 a 6 personas en cada grupo. Se utilizaron técnicas etnográficas tales como observación en la dinámica familiar y notas de campo que contribuyeron a la triangulación de datos. Los lugares de estudio representan las tres zonas geo-socio-territoriales de la Araucanía: costa (lafquenche), valle (nagche), cordillera (pehuenche). Como punto de partida de la selección de lugares y participantes, se ubicó en cada territorio los centros de salud rural con mayor población Mapuche y niños menores de 4 años, luego se contactó al asesor intercultural de cada centro quien facilitó la entrada a las familias, escuelas y agentes tradicionales de la comunidad.

El análisis inductivo de datos se realizó mediante una lectura recurrente de las narrativas (transcripción de entrevistas, grupos focales, registros de observaciones y notas de campo). Se segmentaron los datos en unidades de significado que se codificaron textual y conceptualmente. Se construyó una lista de códigos y se agruparon en unidades similares de significado o dimensiones conceptuales, que sintetizaban los hallazgos del estudio. Surgieron cuatro dimensiones (con sus respectivos códigos) que representaban la forma en que los sujetos de estudio interpretaban y representaban el uso de la lengua indígena en los/as niños/as Mapuche.

La validez de los hallazgos fue acorde al paradigma cualitativo mediante: triangulación de fuentes de información, de técnicas de recolección de datos, y análisis independiente (datos fueron analizados por diversos investigadores). El apego al rigor científico se basó en la coherencia teórico-metodológica; apego a los datos mediante su representación en viñetas, y evaluación externa de su diseño y resultados.

El estudio contó con la aprobación del comité de ética científico de la Universidad de La Frontera, y los participantes firmaron un consentimiento informado que aseguraba la confidencialidad de sus identidades.

\section{Resultados}

Se identificaron 4 dimensiones que caracterizan las prácticas y creencias en torno al uso y estimulación del lenguaje en la población infante Mapuche:

1. Los/as niños/as y el aprendizaje de la lengua representan la mantención de la identidad Mapuche.

2. El habla es un proceso concreto y pragmático.

3. Técnicas tradicionales para estimulación del habla.

4. Dificultades y facilitades para la enseñanza del mapudungun.

\section{Los/as niños/as y el aprendizaje de la lengua repre- sentan la mantención de la identidad Mapuche}

Los niños y niñas Mapuche representan la continuidad de la sociedad y cultura. Ellos tienen la responsabilidad de mantener y continuar el "ser Mapuche" en la sociedad actual. Esta responsabilidad logra mayor relevancia al comprender que el niño o niña representa el futuro y la lengua. Es un sujeto en construcción y formación y por ende una crianza con pertinencia cultural permitirá el traspaso de prácticas, creencias y 
lengua de generación en generación, con el objeto de perdurar la vida Mapuche.

"Sin nuestros niños, nuestro pueblo desaparece. Por eso nosotros insistimos que nuestros niños aprendan nuevamente a hablar nuestro idioma, porque así nosotros vamos a seguir creciendo como lo que somos" (Naña, Puerto Saavedra).

Se valora la importancia de la lengua como una forma de preservar la cultura, el conocimiento y sabiduría Mapuche. Se señala que los niños son más libres en el campo, así aprenden a ser autónomos y libres. Se menciona que desde pequeño se le enseña a ser (che), persona íntegra en idioma Mapuche.

"Nosotros formamos... tiene que ser kimche, norche, zakinche, todo tiene que ser. Tiene que ser una persona buena, amorosa, afectiva, todo eso. Entonces todo eso uno le va diciendo desde esa edad, desde muy pequeño en el campo. Ser libre en el campo, más libertad más autonomía” (Ñaña, Puerto Domínguez).

"Lo que pasa es que a través del habla podemos conservar lo nuestro, que el pueblo sea pueblo, si se pierde el habla se pierde el kimün y el rakizuam, perdemos nuestro horizonte, entonces mientras permanezca seguiremos siendo Mapuche” (Agente ancestral, Ercilla).

\section{El habla es un proceso concreto y pragmático}

Se habla cuando se requiere informar algo, es un acto comunicativo, coherente y con sentido. De otro modo, los niños/as solo requieren observar atenta y silenciosamente; cualidad que se enseña desde muy temprana edad.

"El niño habla cuando es necesario... no por cualquier cosa... debe hablar cuando tiene que hacerlo (...) se le entiende lo que dice y habla fuerte... cosas coherentes $y$ debe tener sentido" (ELCI, Puerto Saavedra).

"Los niños tienen que estar atentos, mirando... viéndolo todo... tienen que estar callados para poder entenderlo todo. Primero observar, no interrumpir... y hablar cuando se le pregunte" (Asesor Cultural, Ercilla).

Se dice que cada niño tiene un ritmo diferente para adquirir el lenguaje. Esto dependerá de su madurez o de la familia en la que vive. Si no aparece el lenguaje a temprana edad, no es visto como problema porque cada niño/a tiene su ritmo.

"Yo siento que está mal, en el sentido que apartamos, separamos, vamos por etapas, es que tal etapa tiene que tener esto o esta habilidad. Pero esas etapas o habilidades se van a desarrollar dependiendo del contexto en el que el niño se desenvuelva. Es todo un proceso que no podemos apurar, que si no cumple esto es un poco retrasado... está mal visto" (Agente ancestral, Temucuicui, Ercilla).

"Hay una edad en que se dice: "ya hablo, yo hablo, soy hablante, yo hablo y me comunico (...) ya a los doce años más menos porque aquí todos los niños dicen a esa edad "yo soy Mapuche" (Ñaña, Puerto Saavedra).

\section{Técnicas tradicionales para estimulación del habla}

El agua que fluye aparece como un elemento que contribuye a la capacidad discursiva. Tomar agua fría entrega vigor, fortaleza y fluidez al lenguaje.

"Tomar agua en la mañana, tres tragos y teníamos que tempranito tomar esa agua, y era tempranito en la mañana y era muy bueno para ser claro para hablar, bien claro (...) tomar agüita corriente de la vertiente ayuda a hablar clarito y de corrido" (Madre, Boroa).

También existen algunas prácticas que ayudan a hablar a los niños cuando observan problemas como caída de la saliva o disfluencia.

"El ave ayuda a comunicarse, un gallina, o la saliva de perro sirve para evitar que se le caiga la saliva al niño". (Ñaña, Curarrehue).

Un mecanismo de estimulación es la observación activa e imitación de sonidos. En diversos relatos se menciona la observación de gestos y conversaciones como mecanismo activo de estimulación del habla, en donde el uso de "kupulwe" (armazón de madera para poner en vertical a los bebés afirmados), permite al niño/a observar el contexto dialógico del acto comunicativo Mapuche.

"Si uno escucha permanentemente, uno escucha y escucha, tu vas a ir aprendiendo, pero ahora nos cambiaron nuestros contextos de vida, al poner a la guagüita en el kupulwe, y las guagüitas paraditas, te miraba la boca $y$ ahora lo ponen en una cuna que no permite ver nada, solo te escucha, está mirando el cielo raso, algo distinto entonces al estar en esta otra posición, no ven como gesticulan" (Asesora cultural, Ercilla).

"Por ejemplo cuando con mi papá salíamos a buscar leña o al monte, y mi papi decía escuchen, y era el sonido del agua (shhhhh) (...) o cuando aparecían los treiles, trugül, trugül, es bien concreto y nos hacía escuchar los pajaritos, uno veía el pajarito y aprendía, primero te lo iban diciendo, por qué finalmente el habla Mapuche viene toda de la naturaleza" (Asesora intercultural, Ercilla). 
La triada "ver, sentir, hacer o practicar", consiste en que el infante observa la situación, siente el sonido, ve como se articula lo asocia, y realiza la acción concreta a la cual estaba expuesto. Dicha acción puede demorarse, no siendo realizada al primer intento, situación por la cual se describe al aprendizaje de la lengua como un proceso persuasivo y comprehensivo.

"Escuchar, ver y hacer, si solo lo escucha no va a comprender, lo mismo si lo ve, eso pasa con las láminas de los chanchos y por eso tiene que ser concreto y visual, por que a través de las imágenes y de lo que están mirando van a aprender $y$ en un contexto real y no imaginario (...) es primordial así es la forma de enseñar, así debería ser en todo pero no se puede y si se da en el colegio estos espacios, hay que aprovecharlo hay que hacerlo" (Agente ancestral, Saavedra).

"Volviendo a lo concreto, si eso, siempre hay que tener los tres, lo auditivo y lo visual y lo hecho" (Agente ancestral, Boroa).

Los relatos, cuentos y consejos son prácticas asociadas a la estimulación de la oralidad y a la comprensión de valores culturales. Los relatos son enseñanzas, cuentan la historia de los territorios, la vida de los antepasados; y la función de la naturaleza.

"Desde la cultura, son los abuelos los que enseñan, más que los papás, siempre están presentes los abuelos, a través de juegos, cuentos (epew), a través de piam (fábulas), se les enseña a los niños como crecer y como ser persona" (Cuidadora, Cholchol).

El canto Mapuche también se usa para ayudar a estimular el habla. En lo dialógico y rítmico los niños/as observan gesticulación, entonación, articulación de los sonidos y modismos de la cultura.

"Por ejemplo si yo le hago ülkantun (canto Mapuche) él se lo aprende muy fácilmente, más que si le enseño frases. El ülkantun es muy, muy importante. El ülkantun ojalá desde muy pequeños le empecemos a hacer el ülkantun, todos los dias... Mientras cocina, mientras lo estamos mudando, mientras lo estamos bañando...". (Cuidadora, Saavedra).

\section{Dificultades y facilitadores para la enseñanza del mapudungun}

Se reconoce la pérdida de la lengua ancestral como una de las principales dificultades para su enseñanza en los/as niños/as. Las generaciones previas (abuelos y bisabuelos) vivieron guerra, persecución y reducción, en donde lo Mapuche fue estigmatizado como atraso ${ }^{13}$.
Dicha vivencia histórica se ha traspasado pasivamente a quienes hoy se encuentran formando una cuarta o quinta generación familiar.

"Hay una pérdida de la lengua... del chezungun (...) hay como una generación que no sabe hablar y menos pueden enseñar (...) yo sufrí mucha discriminación en la escuela por no saber castellano, los profesores (...) los niños se reian" (Agente, Ercilla).

"Nuestro pueblo quedó con una autoestima muy baja y esta presente en todo el pueblo Mapuche, se es parte de un pueblo que fue sometido. Pero los más jóvenes no se creen el cuento" (Cuidador, Curarrehue).

No todos los entrevistados han tenido al mapudungun como una experiencia concreta en su temprana infancia, lo cual ha dificultado su enseñanza en las nuevas generaciones.

"yo creo que no hay quien les enseñe, saben lo básico no más, no hay alguien que les hable todo en lengua. Yo le pregunto a la profe cómo se dice toro, chancho, gallo, pero hay cosas que no saben, no saben todo" (Cuidadora, Curarrehue).

"yo sufrí discriminación en la escuela por no saber hablar el chileno, de ahí yo no le enseñé a mis hijos. Ahora lo estoy recuperando, les estoy enseñando porque también ha habido cambios" (Agente, Ercilla).

Un elemento facilitador de la enseñanza del mapudungun, es que los niños/as vivan en una familia extensa.

La familia grande (...) todos sentados a la mesa (...) $o$ visitando a los parientes que viven aquí, ahí andan los niños (...) ya se les habla y van reconociendo su familia y cómo decir las cosas en Mapuche (Madre, Cholchol).

En algunos territorios, madres y padres jóvenes con alta educación formal, así como también dirigentes sociales, han enfatizado la identidad Mapuche de sus hijos/as desde el nacimiento, realizando algunas ceremonias antes de nacer, seleccionando nombres Mapuche para sus hijos/as, y enseñándoles palabras y cantos en mapudungun.

"yo a mis hijas le pongo nombre en mapudungun, por que a mi no me colocaron. Yo me llamo (...), no me enseñaron mapudungun mis papás, no se por qué, yo fui a un colegio católico entonces, yo no quiero repetir lo mismo con mis hijas, quiero que se sientan orgullosas de ser $\mathrm{Ma}$ puche, que aprendan su lengua" (Madre, Ercilla). 
"En la casa tenemos música e instrumentos, para que ella escuche como se habla, yo le enseño también algunas palabras, como ko, o kofke, asî" (Agente, Boroa).

"Yo le explico que tiene nombre Mapuche y qué significa, que no se tiene que avergonzar de eso" (Cuidadora Cholchol).

Se reconoce que en los últimos años ha habido un mayor interés por enseñar la lengua porque el Estado ha generado una política de educación intercultural en territorios de alta concentración indígena.

"Hay que rescatar lo bueno. Ahora hay profesoras que le enseñan a los niños el Mapuchedungun. Lo están practicando, son ñañas (mujeres) de la comunidad, le dan su comida Mapuche, les enseñan Mapuche. Eso ha ido recuperándose" (Asesora, Saavedra).

"Aquí en el jardin le enseñamos todo lo Mapuche, es intercultural y tratamos que sea bilingüe aunque eso no lo dice el Ministerio que hay que hacer, sólo enseñar la lengua, pero nosotras practicamos toda la cultura, la comida, el canto, el baile, así aprenden a hablar nuestra lengua” (Profesora, Ercilla).

\section{Discusión}

El traspaso y adquisición de la lengua indígena en los niños/as es una preocupación importante en el pueblo Mapuche. Para los entrevistados/as el hecho de adquirir el lenguaje y el acto de habla, ya sea balbucear o representar hechos en el lenguaje, es más que una habilidad o la demostración de un avance en el desarrollo psicomotor de sus hijos. Representa un fenómeno de preservación de la identidad Mapuche. Esto se plantea porque existe en la memoria colectiva de la sociedad múltiples procesos de invasión y colonización que han intentado integrar al pueblo Mapuche a la sociedad chilena, desconociendo su historia, origen territorial y su aporte al proceso de mestizaje de nuestra sociedad. Los relatos también denotan procesos de articulación, recuperación o reivindicación de la lengua indígena en políticas del estado que intentan revitalizar la cultura en aquellos territorios con alta concentración de población indígena.

Existen diversas formas intraculturales para mantener la lengua en la familia tales como, cuentos, consejos, historias, o cantos en mapudungun y que contribuyen a adquirir la lengua Mapuche y los valores de la cultura. Los niños/as saludan en mapudungun, entienden órdenes, reconocen los protocolos culturales. En general, no demuestran al foráneo su bilingüismo, pero como investigadoras observamos la presencia de un código cultural basado en la lengua que niños/as imitan y comprenden. La lengua está viva aún, en pequeños detalles y en formas de comunicación intrafamiliar.

La adquisición del lenguaje como una habilidad psicomotora y cognitiva del infante, no tiene la relevancia intelectual que se le adjudica en nuestra sociedad. Los niños/as hablarán cuando sea necesario, porque hablar es funcional, es para comunicar algo relevante. De otro modo es mejor y valorable observar los acontecimientos de entorno y mantenerse en silencio. En este contexto, aludimos a los trabajos de Bárbara Rogoff quien señala que la observación activa y la imitación juegan un rol fundamental en el aprendizaje de los niños indígenas, más que el explicitar lo que se aprende ${ }^{14}$. Asimismo, Arriaga, Ortega, Meza, et al. señalan que la observación e imitación deben ser considerados como parte del sistema de comportamiento que genera experiencias concretas mediante los sentidos y que se transforma en aprendizaje real ${ }^{15}$. Muy concordante con las palabras de los cuidadores sobre aprender mediante la acción y sensación, Pichon-Rivière señala que sentir, pensar y hacer permiten aprender como un proceso de organizar e interpretar lo observado ${ }^{16}$.

Las conversaciones con familias y profesores nos indican que la adquisición de la lengua es más que decir, nombrar o reconocer cosas; que el niño/a hablará cuando quiera comunicar algo importante; y que esta habilidad depende también de cada niño o niña, de su historia personal o de su linaje. Comunicarse a través de la oralidad es un valor cultural de la persona (ser Mapuche), que está en continuo desarrollo, se logra a través del tiempo, y no es sólo una habilidad cogniti$\mathrm{va}^{17}$.

La adquisición, uso y estimulación del mapudungun en niños y niñas puede considerarse un elemento de resistencia cultural importante para el pueblo Mapuche. Es la entidad simbólica que preserva la identidad y la posibilidad de perdurarse a través de generaciones.

Estudios posteriores dilucidarán temas críticos que se advirtieron en este trabajo pero que por apego a los objetivos no se pudo profundizar, entre estos, el fenómeno de la transgeneracionalidad de los cambios culturales en la enseñanza de la lengua indígena en sus aspectos cognitivos, simbólicos y pragmáticos.

\section{Responsabilidades Éticas}

Protección de personas y animales: Los autores declaran que los procedimientos seguidos se conformaron a las normas éticas del comité de experimentación humana responsable y de acuerdo con la Asociación Médica Mundial y la Declaración de Helsinki. 
Confidencialidad de los datos: Los autores declaran que han seguido los protocolos de su centro de trabajo sobre la publicación de datos de pacientes.

Derecho a la privacidad y consentimiento informado: Los autores han obtenido el consentimiento informado de los pacientes y/o sujetos referidos en el artículo. Este documento obra en poder del autor de correspondencia.

\section{Conflicto de intereses}

Los autores declaran no tener conflicto de intereses.
Financiamiento

FONDECYT 1150833.

\section{Referencias}

1. OMS. [Internet] Cerrando la brecha: La política De acción Sobre los determinantes sociales De La salud, documento de trabajo. [Citado el $10 \mathrm{de}$ mayo de 2019]. Ginebra, Suiza. Octubre 2011. Recuperado a partir de https://www.who.int/sdhconference/ discussion_paper/Discussion-Paper-SP. pdf

2. CEPAL. [Internet] Los Pueblos Indígenas en América Latina: Avances en el último decenio y retos pendientes para la garantía de sus derechos. [Citado el 10 de mayo de 2019] Naciones Unidas. Santiago de Chile, 2014. Recuperado a partir de: https://cpalsocial.org/documentos/71.pdf

3. INE. Resultados CENSO 2017: País, región y comuna [Citado el 10 de mayo de 2019]. Recuperado a partir de: http:// resultados.censo2017.cl/Home/Download

4. Konner M. The Evolution of Childhood: Relationships, Emotion, Mind. 1st ed. Cambrigde, Massachusetts. Belknap Press. 2011.

5. McElroyand A, Townsend P. Medical Anthropology in Ecological Perspective. 6th ed. New York. Routledge. 2015.

6. Alarcón AM, Nahuelcheo Y. [Internet] Creencias sobre el embarazo, parto y puerperio en la mujer Mapuche: conversaciones privadas. Chungará Rev. Antrop. Chil. 40:2 (193-002) [Citado 08 de Mayo 2019]. Recuperado a partir de: https://scielo.conicyt.cl/pdf/chungara/ v40n2/art07.pdf.

7. Sadler M, Obach A, Montecinos M, et al. [Internet] Significaciones, actitudes y prácticas de familias Mapuches en relación a la crianza y cuidado infantil de los niños y niñas desde la gestación hasta los cinco años. Informe técnico, 2006. [Citado 08 de Mayo 2019]. Recuperado a partir de http://www.crececontigo.gob.cl/ wp-content/uploads/2015/11/03-Pautasde-crianza-Mapuche.pdf

8. Quidel J, Pichinao J. Haciendo crecer personas pequeñas en el Pueblo Mapuche. Informe técnico para Seremi Educación Araucanía. Mayo 2002.

9. Gergely G, Bekkering H, Kiraly I. [Internet] Rational imitation in preverbal infants. Nature. 2002. 415(755) [Citado el 9 de mayo de 2019] Recuperado a partir de https://www.nature.com/ articles/415755a.

10. Notari-Syverson A, Losardo A, Lim, Y. Assessment of Young Children from Culturally Diverse Backgrounds: A Journey in Progress. Assess Eff Interv 29(1): 39-51. [Citado el 10 de mayo de 2019] Recuperado a partir de https:// journals.sagepub.com/doi/abs/10.1177/07 3724770302900105?journalCode $=$ aeib.

11. Papalia D, Wendkos S, Duskin R. Psicología del desarrollo: De la infancia a la adolescencia. 11th ed. México. McGRAW-HILL education. 2009.

12. Charmaz I. La Teoría fundamentada en el siglo XXI. Aplicaciones para promover estudios sobre la justicia social. Denzin $\mathrm{K}$, Lincoln Y. Manual de investigación cualitativa, Vol 3: 270-325.

13. Bengoa J. Historia de los Antiguos Mapuches del Sur. Desde antes de la llegada de los españoles hasta las paces de Quilín. 1st ed. Santiago de Chile: Editorial Catalonia; 2007.

14. Rogoff B. Aprendices del pensamiento: el desarrollo cognitivo en el contexto social. 1st ed. Barcelona, España: Paidos ibérica; 1993.

15. Arriaga P, Ortega M, Meza G, et al. [Internet] Análisis conceptual del aprendizaje observacional y la imitación. Rev Latinoam Psicol. 38(1): 87-002. 2006. [Citado el 10 de mayo de 2019]. Recuperado a partir de http://www.scielo. org.co/pdf/rlps/v38n1/v38n1a06.pdf

16. Pichon-Rivière E. El proceso grupal: Del psicoanálisis a la psicología social. 2nd ed. Buenos Aires, Argentina: Nueva visión; 1997.

17. Alarcón AM, Castro M, Astudillo P, Nahuelcheo Y. [Internet] La paradoja entre cultura y realidad: el esfuerzo de criar niños y niñas Mapuche en comunidades indígenas de Chile. Chungará Rev Antrop Chil. 50(4):651-63: 2018. [Citado el 10 de mayo de 2019] Recuperado a partir de https://scielo.conicyt.cl/pdf/ chungara/2018nahead/0717-7356chungara-01601.pdf. 